\title{
Acceleration Performance of Individual European Sea Bass Dicentrarchus labrax Measured with a Sprint Performance Chamber: Comparison with High-Speed Cinematography and Correlates with Ecological Performance
}

\author{
Joshua P. Vandamm ${ }^{\dagger}$ \\ Stefano Marras \\ Guy Claireaux ${ }^{\S}$ \\ Corey A. Handelsman" \\ Jay A. Nelson ${ }^{\#, \dagger}$ \\ Centre de Recherche sur les Écosystemes Marins et \\ Aquacoles, Centre National de la Recherche Scientifique- \\ Institut Français de Recherche pour l'Exploitation de la Mer \\ (CNRS-IFREMER), Place du Séminaire, B.P. 5, 17137 \\ L'Houmeau, France
}

Accepted 4/18/2012; Electronically Published 6/14/2012

\begin{abstract}
Locomotor performance can influence the ecological and evolutionary success of a species. For fish, favorable outcomes of predator-prey encounters are often presumably due to robust acceleration ability. Although escape-response or "fast-start" studies utilizing high-speed cinematography are prevalent, little is known about the contribution of relative acceleration performance to ecological or evolutionary success in a species. This dearth of knowledge may be due to the time-consuming nature of analyzing film, which imposes a practical limit on sample sizes. Herein, we present a high-throughput potential alternative for measuring fish acceleration performance using a sprint performance chamber (SPC). The acceleration performance of a large number of juvenile European sea bass (Dicentrarchus labrax) from two populations was analyzed. Animals from both hatchery and natural ontogenies were assessed,
\end{abstract}

\footnotetext{
* This paper was submitted in response to a call for papers for a Focused Issue on "Intraspecific Variation in Physiology and Behavior."

${ }^{\dagger}$ Present address: Department of Biological Sciences, Towson University, Towson, Maryland 21252-0001.

${ }^{\ddagger}$ Present address: Istituto per l'Ambiente Marino Costiero-Consiglio Nazionale delle Ricerche, Località Sa Mardini, 09170 Torregrande, Oristano, Italy.

${ }^{\S}$ Present address: LEMAR (UMR-6539), Institut Universitaire Européen de la Mer, Technopole Brest Iroise, Plouzané 29280, France.

" Present address: Department of Biology, Colorado State University, Fort Collins, Colorado 80523-1878.

"Corresponding author; e-mail: jnelson@towson.edu.
}

Physiological and Biochemical Zoology 85(6):704-717. 2012. (C) 2012 by The University of Chicago. All rights reserved. 1522-2152/2012/8506-1163\$15.00. DOI: $10.1086 / 666463$ and animals of known acceleration ability had their ecological performance measured in a mesocosm environment. Individuals from one population also had their acceleration performance assessed by both high-speed cinematography and an SPC. Acceleration performance measured in an SPC was lower than that measured by classical high-speed video techniques. However, short-term repeatability and interindividual variation of acceleration performance were similar between the two techniques, and the SPC recorded higher sprint swimming velocities. Wild fish were quicker to accelerate in an SPC and had significantly greater accelerations than all groups of hatcheryraised fish. Acceleration performance had no significant effect on ecological performance (as assessed through animal growth and survival in the mesocosms). However, it is worth noting that wild animals did survive predation in the mesocosm better than farmed ones. Moreover, the hatchery-originated fish that survived the mesocosm experiment, when no predators were present, displayed significantly increased acceleration performance during their 6 mo in the mesocosm; this performance was found to be inversely proportional to growth rate.

\section{Introduction}

Through the integration of morphology with biochemistry and physiology, whole-organism traits such as locomotor performance are believed to influence the ecological success and possibly the fitness of many species (Irschick et al. 2008). In fish, rapid accelerations (fast starts) are believed to play an important role in determining the outcome of predator-prey interactions, particularly for those species participating in "sit-and-wait" predator-prey interactions (Domenici and Blake 1997; Walker et al. 2005). A fast start is considered the unsteady (transient) motion that results from the first contraction of the lateral musculature, typically lasting less than $1 \mathrm{~s}$, of which only the first $50 \mathrm{~ms}$ is generally analyzed (Domenici and Blake 1997). Numerous studies have reported fast-start swimming speeds that greatly exceed maximal swimming speeds recorded during graded interval swimming tests (Marras et al. 2010). Although absolute swimming speed may be a determining factor in predator-prey encounters that occur over meters and seconds, acceleration is more likely to be the critical factor when encounters transpire over centimeters or milliseconds (Walker et al. 
2005; O'Steen et al. 2010). Despite the tremendous amount of information about sensory integration, neural control, biomechanics, kinematics, muscular power output, hydrodynamics, and whole-organism acceleration performance during fish fast starts (e.g., Johnston et al. 1995; Blake and Law 1996 Domenici and Blake 1997; Wakeling and Johnston 1998; Domenici 2001; Eaton et al. 2001; Wakeling 2001; Hale et al. 2002; Tytell and Lauder 2008), little is known about the contribution of relative acceleration performance to ecological or evolutionary success in fishes. One reason for this may be the time-consuming nature of studying acceleration by traditional methods. Usually, fast-start analyses involve high-speed cinematography and frame-by-frame analysis, often with only a single trial per study animal and relatively small sample sizes (typically 10-40 animals per study). With such a small sample size, the extent of interindividual variation may not be fully represented, and any subsequent tests of the ecological or evolutionary relevance of acceleration performance become questionable. To adequately examine the role of acceleration performance in ecological or evolutionary outcomes, techniques that measure acceleration rapidly and repeatedly on large numbers of animals under realistic conditions are thus required (Kingsolver et al. 2001).

Previously, a sprint performance chamber (SPC; Nelson et al. 2002) has been used to assess maximal swimming speed of European sea bass (Dicentrarchus labrax) during a burst swim initiated by an attempted grasp of a human hand (Nelson and Claireaux 2005; Claireaux et al. 2007; Handelsman et al. 2010). Swimming speed measured by this technique uncovered substantial intraspecific variability in similar-sized animals and was found to be significantly repeatable on a daily basis and across 4 wk including a temperature transition (Nelson and Claireaux 2005; Claireaux et al. 2007). With this technique, wild European sea bass were found to have higher sprint swimming speeds than cultured fish and survived natural predation better (Handelsman et al. 2010). Moreover, under simulated natural conditions, sprint swimming speed was negatively correlated with somatic growth. What proportion of these findings can be attributed to the "fast-start" component of a burst swim and whether the substantial intraspecific variation and repeatability found for burst swimming performance (Nelson and Claireaux 2005; Claireaux et al. 2007) also hold for acceleration performance have not yet been determined.

That acceleration performance is subject to mortality selection in nature is generally assumed but rarely demonstrated. For a performance trait to be under selection, it must (1) be a heritable determinant of fitness, (2) vary among individuals, and (3) possess demonstrable repeatability (Endler 1986; Falconer and Mackay 1996; Dohm 2002; Oufiero and Garland 2009; Marras et al. 2011). Similarly, for a performance test to be useful in studying organismal ecology or fitness, it should mimic challenges to the phenotype that are experienced in the wild during a comparable life-history stage (Nelson et al. 2002). Unfortunately, there is only limited evidence for the repeatability of fast-start performances in fish (e.g., Gibson and Johnston 1995; Fuiman and Cowan 2003; Oufiero and Garland 2009;
Marras et al. 2011), and we are unaware of any studies tracking the relative fortunes of individual fish of known acceleration performance in the field. Several studies have gauged the success of animals of known acceleration performance subjected to predator-prey encounters but only under laboratory conditions (e.g., O'Steen et al. 2002, 2010; Chappell and Odell 2004; Walker et al. 2005). Our study extends this type of study to the seminatural mesocosm level.

This study was conducted to build on current knowledge concerning the repeatability and ecological relevance of acceleration performance in juvenile fish. Our first objective was to evaluate the use of an SPC for measuring the interindividual variance and daily repeatability of acceleration performance in a highly motile fish species, the European sea bass, and to compare the acceleration values obtained with those from traditional high-speed cinematography. A second objective was to compare the acceleration performance of juvenile sea bass reared in fish culture facilities with conspecifics captured directly from the wild. Aquaculture of European sea bass is extensive throughout Europe, and escape from farming operations is commonplace (Toledo-Guedes et al. 2011). These escaped fish could potentially dilute the gene pool of natural populations with genes that are favored in culture but are selectively disadvantageous in the wild (Brannon et al. 2004). Comparing acceleration performances of wild and cultured fish could provide information useful for assessing the wisdom of cultured fish introductions to sustain natural populations and for best management practices of the aquaculture industry. By transferring experimental populations to seminatural mesocosms, our final objective was to evaluate the selective value of acceleration performance by testing whether acceleration measured with an SPC correlated with proxies for fitness such as growth and mortality.

\section{Material and Methods}

\section{Fish Collection and Maintenance}

Cultured Fish. Juvenile European sea bass of both sexes examined in this study were collected from two different farming operations in France: 165 fish were obtained from a hatchery in Ferme Marine des Baleines, Ile de Ré (Atlantic Ocean), and 37 fish were obtained from Extramer fish farm in Salses le Chateau (Mediterranean Sea). The Atlantic broodstock was four generations removed from the wild and had a $10 \%$ annual renewal rate. The Mediterranean stock was captured as small juveniles and raised in the culture facility. Atlantic Ocean fish were transported to the Centre de Recherche sur les Ecosystèmes Marins et Aquacoles (CREMA) in L'Houmeau, France, for experiments. Mediterranean Sea fish were experimented on in the Station Méditerranèenne de l'Environnement Littoral in Sète, France. Atlantic fish were juveniles (length: $\bar{X}=16.8 \pm$ $1.4 \mathrm{~cm}$ [mean $\pm \mathrm{SD}$ ]; range, $13.6-21.3 \mathrm{~cm}$; mass: $\bar{X}=$ $46.2 \pm 13.4 \mathrm{~g}$ [mean $\pm \mathrm{SD}$ ]; range, $21.4-89.9 \mathrm{~g}$ ), as were the Mediterranean fish (length: $\bar{X}=16.3 \pm 1.2 \mathrm{~cm}$ [mean $\pm \mathrm{SD}$ ]; range, $13.8-18.4 \mathrm{~cm}$; mass: $\bar{X}=56.8 \pm 10.7 \mathrm{~g}$ [mean $\pm \mathrm{SD}$ ]; range, $34.9-77.8 \mathrm{~g}$ ). Fish were distributed among 500-L tanks 
supplied with recirculating filtered natural seawater $\left(28 \%{ }^{-}\right.$ $32 \%$ at $20^{\circ} \mathrm{C}$; renewal rate of $30 \%-50 \%$ per week). Natural photoperiod conditions were maintained. Fish were fed three to four times per week with commercial dry pellets (Bar D PerformNatura 4.5, Sica du Gouessant, Lamballe, France). Feedings were discontinued $24 \mathrm{~h}$ before any handling of the animals or a performance trial. Fish were acclimated to laboratory conditions for at least 1 mo before experimentation started. At least 1 wk before experimentation, fish from both populations were anesthetized (2-phenoxyethanol; dilution 0.3 $\mathrm{mL} / \mathrm{L})$; weighed $(\mathrm{g})$; measured for length, width, and depth $(\mathrm{cm})$; and subcutaneously tagged behind the dorsal fin with a passive integrated transponder (PIT-Tag, Ordicam, Rambouillet, France).

Wild Fish. Sixty-two wild juvenile fish $15.6 \pm 2.7 \mathrm{~cm}$ in length (range, 12.9-18.3 cm) and 33.1 $\pm 17.7 \mathrm{~g}$ in weight (range, 15.4$50.8 \mathrm{~g}$ ) were captured by beach seine off the northeastern shore of Ile de Ré, France, over 3 wk in June and transported to the nearby CREMA laboratory. Handling and tagging procedures were identical to those for cultured fish. The fish handling protocol was approved by Towson University's Institutional Animal Care and Use Committee (F9900RR.08) and conformed to French government standards and regulations.

\section{Acceleration Performance}

Sprint Performance Chamber and Fish Handling. The SPC was modeled after that described by Nelson et al. (2002). This newer version employed more advanced electronic technology, and the dimensions were scaled for juvenile Dicentrarchus labrax. It is described in detail in Nelson and Claireaux (2005). Briefly, dimensions of the chamber were $2.00 \mathrm{~m}$ (length) $\times 0.25 \mathrm{~m}$ (width) $\times 0.30 \mathrm{~m}$ (height). Eight parallel 5-mW light-emitting laser diodes of 645-670-nm wavelength and their corresponding detector arrays were placed at staggered intervals of $0,0.02$, $0.04,0.08,0.16,0.41,0.66$, and $0.91 \mathrm{~m}$ from the starting line. The first three intervals of 2,2 , and $4 \mathrm{~cm}$ were specifically designed for measurements of acceleration. Within each detector array, sensors were placed every $1.5 \mathrm{~cm}$, beginning $1 \mathrm{~cm}$ from the bottom and proceeding vertically to a height of 13 $\mathrm{cm}$ (1 $\mathrm{cm}$ below the water surface). The deliberately shallow 14-cm depth helped limit vertical swimming trajectories. The $1.5-\mathrm{cm}$ separation distance was empirically determined (based on juvenile sea bass morphology) to guarantee that a detector would be triggered within the first centimeter of a passing fish. A dark area of the chamber immediately before the starting line simulated natural cover, coaxing fish to voluntarily orient themselves and remain relatively motionless before a trial began. Once oriented, fish were startled by hand in an attempt to mimic a predator and chased down the drag strip to encourage maximal swimming performance. Each fish sprinted four to six times and was given at least 5 min recovery time with no human contact between each trial. Speed was calculated by dividing the distance between intervals by the time between corresponding beam breakages. Acceleration was further de- termined by the difference in speed of a subsequent interval divided by the time elapsed between intervals, summarized by the equations

$$
\begin{aligned}
& v=\frac{\Delta x}{\Delta t}, \\
& a=\frac{\Delta v}{\Delta t},
\end{aligned}
$$

where $a$ is acceleration $\left(\mathrm{m} \mathrm{s}^{-2}\right), \Delta t$ is time elapsed between subsequent detector array activations $(\mathrm{s}), v$ is speed $\left(\mathrm{m} \mathrm{s}^{-1}\right)$, and $\Delta x$ is the distance $(\mathrm{m})$ between detector arrays. False detections were usually apparent during data analysis, and because such large amounts of data were produced, any suspect recordings were not used. Acceleration values were calculated only from velocities that were within $0.5 \mathrm{~m} \mathrm{~s}^{-1}$ of a value from a separate trial. Likewise, an acceleration value was considered valid only if there was another value from the same fish from a separate trial within $150 \mathrm{~m} \mathrm{~s}^{-2}$ of that value. Only fish that had at least three good trials were included in the analysis. This resulted in usable data from 95 cultured fish from the Atlantic population, 38 of which were retested after surviving 6 mo in a mesocosm (see below); a separate group of 37 cultured fish from the Atlantic population, all of whom perished during a 14-wk stay in mesocosms (see below); 62 wild juvenile sea bass from the Atlantic population, only 40 of which were released for a 14-wk stay in mesocosms; and 37 fish from the Mediterranean population that were also filmed for fast-start analysis (see below). With four to six trials per fish $(\bar{X}=5.5)$, a total of approximately 1,480 sprint trials are reported on here.

Electronics. Light-emitting laser diodes $(5 \mathrm{~mW}, \lambda=645-670$ $\mathrm{nm}, 1.1-\mathrm{mm}$ beam width) were purchased from OnPoint Lasers (Eden Prairie, MN) and Selectronic (Lille, France). The 72 photo Darlington detectors (nine sensors/array, eight arrays; detection $\lambda$ of $580-720 \mathrm{~nm}$ ) were manufactured by Honeywell International (Morristown, $\mathrm{NJ}$ ). When activated by light, the photo Darlington detector array puts out a $5-\mathrm{V}$ signal to one of eight inputs on a Daqboard 200 data acquisition board (Iotech, http://www.iotech.com) inside a Windows-compatible computer. When all nine sensors in an array are saturated with light, the output is a constant $5 \mathrm{~V}$. However, disruption of the light path to any one sensor of an array causes the output from the entire array to drop below $1 \mathrm{~V}$. When armed, the computer scans the first array at a rate of $19.2 \mathrm{kHz}$ and begins collecting data on breaking of the first light beam by a fish. Data were assimilated using Daqview software (Iotech), utilizing a proprietary Labview (National Instruments, http://www.ni.com) routine (G. Claireaux, unpublished data) but can be assimilated with commercial analog to digital units.

High-Speed Camera Measurements. The experimental setup was made up of a circular tank (100-cm diameter $\times 80$-cm depth and $25-\mathrm{cm}$ water depth) supplied with recirculating seawater at $20^{\circ} \mathrm{C}$. The escape response of the fish was induced by mechanical stimulation. The stimulus was a PVC cylinder with a 
tapered point and an iron bolt at the opposite end $(10-\mathrm{cm}$ height, 2-cm diameter, and weighing $35 \mathrm{~g}$ ). The stimulus was released by an electromagnet from a height of $150 \mathrm{~cm}$ above the water surface. To prevent visual stimulation before contact with the water surface, the stimulus was released into a vertical PVC tube ( $15 \mathrm{~cm}$ in diameter) ending $0.5 \mathrm{~cm}$ before the water surface (Lefrançois and Domenici 2006). Light was supplied by two $250-\mathrm{W}$ spotlights, and the whole setup was covered by a black tarpaulin to screen the fish from visual disturbance. A high-speed camera (Red Lake Motion Scope) was positioned above the experimental tank. It was connected to a Windowscompatible computer by a dazzle system and recorded the escape response at $250 \mathrm{~Hz}$. The camera was triggered to record from $1 \mathrm{~s}$ before the stimulation to $3 \mathrm{~s}$ after the stimulation. Two-dimensional $x$ - and $y$-coordinates of the fish's center of mass $(\mathrm{CM})$ and tip of the head were plotted every $4 \mathrm{~ms}$ from $20 \mathrm{~ms}$ before to $160 \mathrm{~ms}$ after the stimulus onset (45 frames in total). Fish were tested three times with 30 -min intervals between successive startles; maximum escape acceleration $\left(\mathrm{ACC}_{\max }\right)$ was taken to be the fastest value achieved during the three tests.

\section{Ecological Performance}

CREMA-L'Houmeau's tidal earthen pond $\left(200 \mathrm{~m}^{2}\right.$ and $\sim 1 \mathrm{~m}$ deep) facilities were used to test the ecological performance of three of the groups of juvenile D. labrax. These ponds connect to the Atlantic Ocean (Bay of Biscay) via a tidal canal, allowing a natural forage base to arrive with each incoming tide, while standpipes and meshing prevent the experimental fish from escaping (Handelsman et al. 2010). Fish were able to forage, compete, and evade predators in an environment similar to what they would be exploiting at this life-history stage in nature (Pickett and Pawson 1994). These ponds also permitted periodic recovery of fish for assessment of mortality and growth rate.

Fish from two separate experimental releases are reported on here (Handelsman et al. 2010). After the laboratory measurements were completed, cultured D. labrax juveniles were either (1) released at densities that ensured intraspecific competition for food (60 fish per pond) but were free from predation or (2) released at much lower densities (20 fish per pond) but exposed to avian predation. Previous research had established that for fish and ponds of this size, density begins to limit growth at 30 fish per pond (Handelsman et al. 2010). For the first experiment, animals were in the simulated estuaries for $24 \mathrm{wk}$ and were removed to assess survivorship and growth at weeks 8 and 16. At the 16-wk collection, mortality had approached $50 \%$ and all surviving individuals had lost mass, so the decision was made to supplement the food in each pond with equal volumes of live, natural food seined from two adjacent artificial estuaries without fish and distribute it equally between the two experimental estuaries. Surviving fish were recaptured from the earthen ponds and brought back to the laboratory for a 5-wk acclimation period, after which 38 of them were sprinted again to examine long-term repeatability of acceleration performance and the influence of growth. In the second release, juveniles were randomly selected without any knowledge of performance but were allocated to achieve equal biomass in each pond and kept for $14 \mathrm{wk}$ in the mesocosms during the late summer and early autumn. The ability to evade predators during this time was tested by allowing local populations of Ardea cinerea (gray herons), Egretta garzetta (little egrets), and Phalacrocorax carbo (great cormorants) unrestricted access to the estuaries (Handelsman et al. 2010).

\section{Statistics}

Analyses were performed using Statistica 8.0 for Windows and Statistica 5.0 for Macintosh. The fiducial level of significance was 0.05. Nonparametric Spearman rank and Kendall concordance coefficients were used to determine intraindividual repeatability of performance. ANCOVA with mass as the covariate was employed to evaluate variation between groups, and $\mathrm{Tu}-$ key's HSD test was used to distinguish among the groups within a significant ANCOVA test. Kolmogorov-Smirnov tests were used to evaluate the normality of distributions, and Fisher's exact test was used to determine whether distributions were significantly different. Multiple least squares regressions were used to evaluate the relationship between acceleration performance, fish morphometrics, and growth rate. Differences between tests made on the same individual were assessed with a paired $t$-test. The $\chi^{2}$ test was used to assess the independence of trial order.

\section{Results}

Efficacy of Acceleration Determinations and Comparisons with Film

Because the velocity of a fish is unknown as it breaks the first laser in the SPC, the investigator is faced with uncertainty in calculating an animal's maximal acceleration. Taking the fish's initial velocity as 0 will artificially inflate acceleration values because the fish had to be moving to break the first laser beam. However, not using the first interval will fail to incorporate the animal's initial fast start and thus be unlikely to capture its maximal acceleration. Even though trials were initiated only when the fish was oriented with the tip of its head pointed down the chamber, there is no assurance that the initial phases of a fast start are being captured with an SPC. In the following report, we have opted for the more conservative approach of not using the first 2-cm interval of the SPC in our maximal acceleration analysis. However, we first offer the reader a short analysis of the consequences of not using this first interval. We do this through a comparison of accelerations of the wild fish, calculated either with or without the first interval (fig. 1). In addition, we compare accelerations of the Mediterranean fish, calculated either from traditional high-speed cinematography or from the SPC without the first interval (fig. 2).

Figure 1 demonstrates that incorporating the first laser interval into the acceleration computation produces values for maximal acceleration that are much greater and more variable 

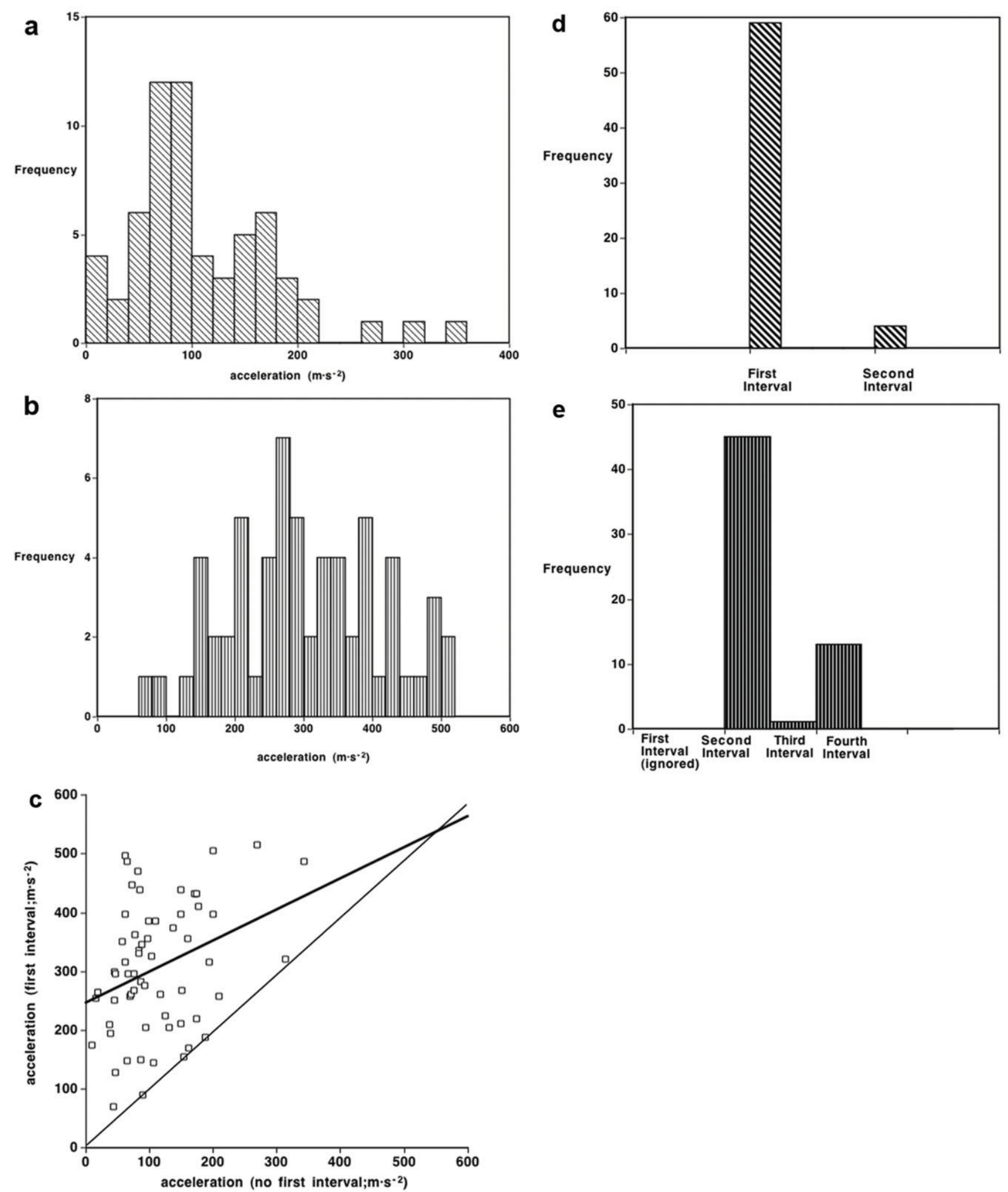

Figure 1. A comparison of acceleration data analysis methods for only the cohort of wild-caught European sea bass measured in the sprint performance chamber (SPC). Histograms of the top acceleration measured by each method: $(a)$ not including the first $2-\mathrm{cm}$ interval and $(b)$ including the first $2-\mathrm{cm}$ interval. The graph in $c$ is a direct comparison of the two methods with a best-fit least squares regression (thick line: ACC $($ first interval $)=0.532 \times$ ACC (no first interval) $+246 ; r^{2}=0.11$ ). The thin line is the line of identity. The final two histograms are of the laser interval on the SPC that produced a top acceleration value for each fish: $d$ includes the first interval, and $e$ excludes it. 



and that better conform to a normal distribution than if excluded (fig. 1a-1c). This figure also shows that if the first interval is included, a fish's maximal acceleration is almost exclusively recorded from this interval (fig. 1d). In contrast, when the first interval is excluded, the maximal acceleration is generally recorded from the second $2-\mathrm{cm}$ interval but not as exclusively (fig. 1e). Interestingly, an animal's maximal accelerations with or without inclusion of the first interval were only weakly correlated $\left(\mathrm{ACC}_{\max 1 \mathrm{st}}=0.53 \times\left(\mathrm{ACC}_{\max \text { no 1st }}\right)+246\right.$; $R^{2}=0.11$; fig. 1 c). This result is also reflected in differential assessments of repeatability when comparing the two different analyses. If the first interval is included in the analysis, comparing an animal's top acceleration performance with its second best does not indicate significant repeatability (Spearman $r=0.162, P=0.22$; Kendall $\tau=0.126, P=0.16$ ). However, if the first interval is excluded from the analysis, acceleration measured with the SPC is now very repeatable (second-best vs. third-best performance of previous analysis; Spearman $r=$ 0.531, $P<0.001$; Kendall $\tau=0.415, P<0.001$ ).

Maximal accelerations measured in the SPC (excluding the first interval) were poorly correlated with maximal accelerations measured in the same fish with a high-speed camera $\left(\mathrm{ACC}_{\operatorname{max~cam}}=0.3 \times\left(\mathrm{ACC}_{\max \text { no 1st }}\right)+110 ; R^{2}=0.02\right.$; fig. 2a) and were significantly lower $(P<0.001$, paired $t$-test; fig. $2 b)$. Higher swimming velocities were recorded with the SPC than with the high-speed camera $(P<0.001$, paired $t$-test; fig. $2 c)$, but when comparing an animal's fastest acceleration with its second-fastest acceleration, the two techniques had almost identical repeatability (camera: Spearman $r=0.606$; Kendall $\tau=0.483, P<0.001$; SPC: Spearman $r=0.606$; Kendall $\tau=$ $0.467, P<0.001$; fig. $2 d$ ).

For all but one group of fish, an individual's maximum acceleration was independent of trial order $\left(\chi^{2}\right.$ test, $\left.P>0.2\right)$, mirroring previously published results for sprint speed (Handelsman et al. 2010) and suggesting that juvenile Dicentrarchus labrax generally displayed no learning curve or signs of exhaustion as they proceeded through the four to seven multiple trials. For the cultured fish from the Atlantic population that were released to the marshes alongside the wild fish (second release), $76 \%$ of the fish had their maximum acceleration recorded in one of the first three trials, producing a significantly nonrandom trial effect $\left(\chi^{2}=17.23, P<0.01\right)$.

Figure 2. A comparison of data obtained from individuals of the Mediterranean population by either chasing in a sprint performance chamber (SPC) or high-speed filming after being startled by a simulated predator. $a$, Direct comparison of the two methods for each individual fish; SPC is on the abscissa and cinematography on the ordinate: camera $=0.296 \times \mathrm{SPC}+110.036, r^{2}=0.017 . b$, Mean population values \pm 1 SEM for acceleration estimated by each method. $c$, Mean population values $\pm 1 \mathrm{SE}$ for top swimming speed estimated by each method. $d$, Daily repeatability of the two methods. Each animal's top performance for that day is plotted on the abscissa and the secondbest performance for that day on the ordinate with the best-fit least squares regression plotted; filled diamonds and solid line $=\mathrm{SPC}(\mathrm{sec}-$ ond $)=0.56 \times$ first $+1.95\left(r^{2}=0.5\right)$; open squares and dotted line $=$ cinematography $($ second $)=0.44 \times$ first $+46.3\left(r^{2}=0.22\right)$. 
Maximum rates of acceleration from a single interval from each of an individual's three best trials were analyzed. Within all groups of fish, the highest rates of acceleration were most likely to be recorded in one of the first three intervals of 2,4 , and $8 \mathrm{~cm}$, respectively (in this analysis, the initial $2-\mathrm{cm}$ interval was not analyzed; see above; fig. 1). Fish from all of the groups were most likely to reach their peak acceleration in the second $2 \mathrm{~cm}$ of the SPC; however, the percentage of wild fish that did this $(76 \%)$ was greater than for any of the groups of cultured fish $(57 \%-63 \%)$. The maximum speed reached by a fish (Claireaux et al. 2007; Handelsman et al. 2010) was a significant but weak function of a fish's best acceleration performance (ANOVA, $P<0.01$; fig. 3 ), although each measurement was not necessarily from the same trial or interval.

\section{Effects of Size and Ontogeny}

Mass had no significant effect on acceleration within any single group of fish, but mass-acceleration regressions of individuals from all five groups together indicated a significant negative relationship. According to this large data set $(n=272)$, acceleration best scales linearly and negatively with mass, albeit weakly $\left(r^{2}=0.043\right.$; ACC $=-0.591 M_{\mathrm{b}}+93.6 ; P<0.001$; when mass is measured in $\mathrm{g}$ and acceleration in $\mathrm{m} \mathrm{s}^{-2}$ ). The negative scaling of acceleration with mass is predictable from first principles (Vogel 2008). An ANCOVA with fish mass as the covariate revealed significant intergroup variation in acceleration $(P<$ 0.0001 ), and post hoc analysis showed that wild fish accelerate significantly faster as measured in an SPC than do all groups of cultured fish (Tukey's $P<0.0001$; fig. 4). Similarly, despite fish growth in the mesocosms and the negative scaling of acceleration with size and growth (see below), cultured fish that survived the mesocosms without predation had significantly better acceleration than the cultured fish from the Mediterranean (Tukey's $P<0.01$; fig. 4 ). A repeated-measures ANCOVA of just those cultured fish that survived the mesocosms revealed that their postmesocosm acceleration surpassed their initial acceleration $(P<0.001 ; 31 / 38$ fish improved $)$, suggesting that surviving the mesocosms entailed improvement of acceleration performance and not directional selection for those animals that had initially high performance.

Food supplementation during the last interval of the first release (see above) allowed the remaining fish to achieve the highest growth rates of the experiment during the final $8 \mathrm{wk}$ in the two estuaries. Maximum acceleration measured in the SPC was negatively related to growth rate in those animals that survived the mesocosms but only if condition factor was incorporated as a covariate (ANOVA, $P<0.05$ ). The best-fit relationship for acceleration performance as a function of just growth rate was a negative exponential function (fig. 5).

\section{Repeatability and Variation}

As reported previously for maximal sprint speed measured in the same sprint chamber (Claireaux et al. 2007), interindividual variability in acceleration performance exceeded intraindividual variation in consecutive trials, demonstrating short-term repeatability for all groups (table 1). This significant consistency in individual acceleration performance supports the provisional use of the sprint chamber for evolutionary and ecological studies, although acceleration performance in cultured fish after 6 mo in the mesocosms was not repeatable (Spearman rank $r=0.138, P=0.41$; Kendall $\tau=0.081, P=0.47)$. This lack of repeatability is probably due to the profound phenotypic reshuffling undergone by developing juvenile fish in the mesocosms, where they experience growth and multiple changes

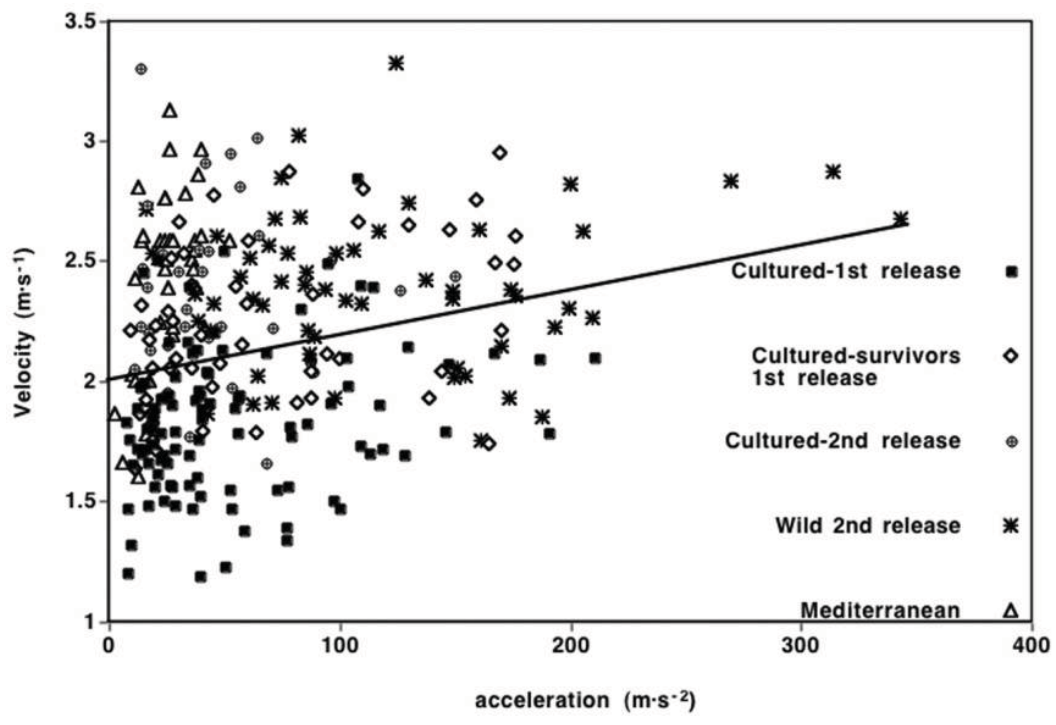

Figure 3. Top swimming speed reached by a fish as a function of its top acceleration by population and cohort. The best-fit least squares line is plotted: maximum velocity $\left(\mathrm{m} \mathrm{s}^{-1}\right)=0.002 \times \mathrm{ACC}_{\max }+2.06 ; r^{2}=0.045 ; P<0.001$. 


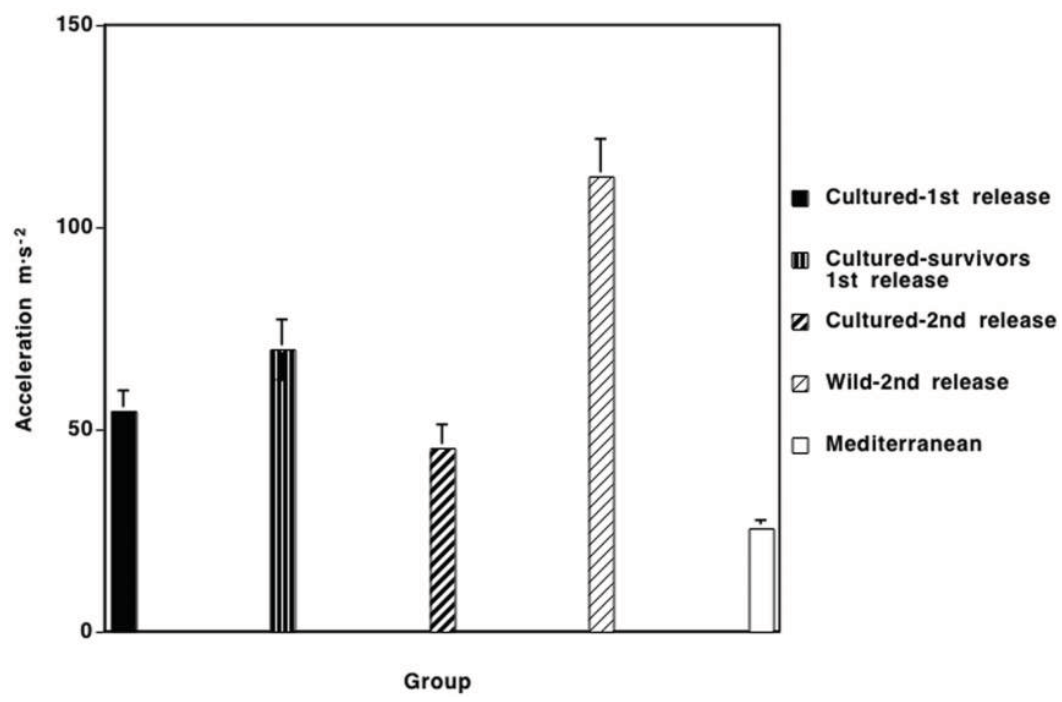

Figure 4. Mean top acceleration by population and cohort \pm 1 SE. All filled bars are from the Atlantic population; the unfilled bar represents the Mediterranean population. Least squares means are plotted in accordance with the significant covariate of mass, but because of the small size range, these were never more than $2 \%$ different from the actual mean.

in environmental conditions. This result mirrors the lack of 6mo repeatability in maximal sprint speed measured in the same sprint chamber (Claireaux et al. 2007). Top accelerations were significantly normally distributed except for the cultured firstrelease group, which was significantly lognormally distributed. The coefficients of variation for top acceleration were substantially greater than for velocities measured in this chamber (Handelsman et al. 2010) and were substantially lower for the wild fish and the cultured Mediterranean groups (table 1).

\section{Mesocosm Performance}

Laboratory measurements of acceleration performance were not predictive of survival of cultured fish in the mesocosms under high-density conditions without predation. The factors that best predicted survival of cultured fish were growth rate during the previous interval and condition factor of the fish at the onset of the interval (Handelsman et al. 2010). There was also no significant effect of acceleration on growth rate during any interval or across the entire 6 mo of mesocosm residence for cultured fish, although growth rate did negatively impact acceleration performance after removal from the marshes (fig. $5)$.

In the second release, no cultured fish survived $14 \mathrm{wk}$ in the artificial estuaries exposed to avian predation, whereas 14 wild fish $(35 \%$; 8 in one estuary, 6 in the other) survived (Handelsman et al. 2010). Because the wild fish differed significantly from cultured fish in a number of parameters (ontogenetic history, length, mass, maximal sprint speed, and maximal acceleration performance), it is difficult to ascribe the differential survival of wild fish to any one factor. The wild fish that survived $14 \mathrm{wk}$ in the artificial estuaries exposed to predation
( $n=14)$ had marginally greater sprint capacity than those that perished ( $n=26$; Handelsman et al. 2010), but the 12 survivors that conformed to our selection criteria for analyzing their acceleration performance did not have significantly greater acceleration performance when they entered the mesocosms than did the wild fish that perished.

\section{Discussion}

Efficacy and Future of Acceleration Determinations with an SPC

A major challenge in analyzing acceleration data from the SPC related to the fact that swimming speed was not 0 when fish broke the first detector array. Faced with this difficulty, the question was whether to include or reject data from the interval between the first and second detector arrays. To include this first interval meant assuming that the fish had 0 velocity as it broke the initial beam, which, by definition, is not true. However, to exclude this interval meant eliminating some of an animal's fast-start performance from the analysis, undoubtedly compromising estimates of the animal's maximal acceleration capability (Domenici and Blake 1997). We chose to report on acceleration performances exclusive of this first interval, although we do present a comparison of both analysis options for one population (fig. 1).

The presumed major consequence of not including the first interval in the analysis is that lower-than-maximal accelerations were recorded. This suspicion was confirmed not only by the lower values in wild fish analyzed both ways (cf. fig. 1a, 1b) but also through a comparison of the same individuals filmed both undergoing a fast start and chased in an SPC (fig. 2). Estimated maximal accelerations were consistently lower in the 


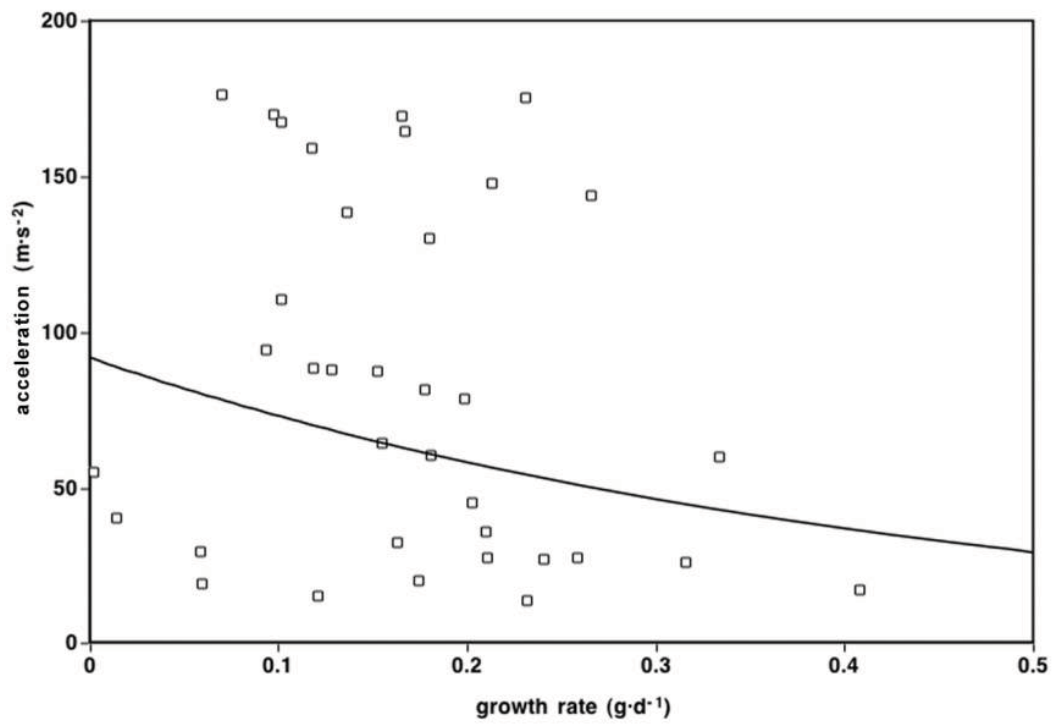

Figure 5. Top acceleration of 52 cultured juvenile sea bass from the first release to the mesocosms as a function of their growth rate during their last interval in two experimental estuaries when forage was abundant and densities were low. The best-fit declining exponential function is also depicted: maximum acceleration $\left(\mathrm{m} \mathrm{s}^{-2}\right)=91.6 \times 10^{-0.98 \times \text { growth }}$.

SPC, but repeatability and variance of acceleration measurements were similar between the two methods (fig. 2). Had we included the first interval in our estimates of maximal acceleration from the SPC, we would have reported higher estimates of acceleration capacity but coincident with greater variance and nonsignificant repeatability. In future use, investigators will have to weigh the respective benefits and liabilities of using an SPC and incorporating the first interval before adopting an SPC for estimating acceleration capabilities of aquatic organisms in ecological and evolutionary studies. Invention of a contrivance that motivates the animal to orient directly at the starting line, without restricting movement or inducing stress, could make the SPC the method of choice for measuring acceleration performance in large groups of aquatic animals. $\mathrm{Al}-$ ternatively, an SPC that kept animals at a constant low-flow velocity before motivating them to accelerate, thereby standardizing initial velocity, would be an important advance.

Nevertheless, measurement of acceleration by juvenile sea bass with an SPC produced individually repeatable performances with substantial variation between conspecific individuals. This finding opens the door for use of this method to study the ecological and evolutionary relevance of variation in acceleration performance by fishes. The concept of using an SPC for determining acceleration is described in Nelson et al (2002), but this is the first systematic examination of a substantial number of individuals from the same species with the method. Acceleration values measured here, while acknowledged underestimates, compare favorably with those generated through traditional high-speed cinematography $\left(80 \mathrm{~m} \mathrm{~s}^{-2}\right.$ for 26.5- $\mathrm{cm}$ farmed fish; Lefrançois and Domenici 2006; $90 \mathrm{~m} \mathrm{~s}^{-2}$ for 12-cm, 17-g wild fish; S. Marras et al., personal commu- nication) and were statistically correlated with factors such as growth rate and the location where ontogeny occurred.

With the exception of one group, European sea bass had an equal probability of having their maximum acceleration measured in any of their four to seven trials (not shown). For the single cultured population that did not adhere to this finding, later trials were underrepresented in recording maximum acceleration events, suggesting that fish were either becoming fatigued or habituating as the trials progressed. This was different from the results obtained for maximal sprint velocity in an SPC over $25-\mathrm{cm}$ intervals, wherein a slight tendency to improve in consecutive trials was observed (Nelson and Claireaux 2005) or no time effect was observed (Handelsman et al. 2010). The group of fish that showed a declining probability of maximal acceleration being recorded after trial 3 had been in the laboratory for a relatively long period $(\sim 4+$ mo) before being chased in the SPC, so habituation to humans is our most likely explanation for this result. Fatigue is unlikely because no other group of cultured fish exhibited a similar trend and all were housed and fed identically and appeared equally healthy.

If one considers an equal probability of measuring the maximum acceleration in any given trial and our mean number of trials per fish of 5.5 , we estimate that we had an $18 \%$ chance of underestimating the acceleration performance of any given fish. Adolph and Pickering (2008), working with the lizard Sceloporus occidentalis, demonstrated that maximum performance measurements increase with trial number, with five trials yielding an $89 \%$ average estimate of the true maximum, observed after 20 trials. Based on the results of Adolph and Pickering (2008), as well as our own, we recommend a minimum 
Table 1: Sample sizes, mean acceleration as measured with the sprint performance chamber, two measures of repeatability, and the coefficient of variation for the acceleration estimates for each population and cohort

\begin{tabular}{lccccc}
\hline Group & $n$ & $\begin{array}{c}\text { Mean acceleration } \\
\left(\mathrm{m} \mathrm{s}^{-2}\right)\end{array}$ & Spearman $r$ & Kendall $\tau$ & $\begin{array}{c}\text { Coefficient of } \\
\text { variation (\%) }\end{array}$ \\
\hline Cultured first release & 99 & 55.8 & .686 & .520 & 80.4 \\
Survivors first release & 52 & 68.1 & .724 & .556 & 79.5 \\
Cultured second release & 38 & 45.1 & .764 & .566 & 70.9 \\
Wild second release & 59 & 115.7 & .531 & .415 & 59.9 \\
Mediterranean & 37 & 25.6 & .606 & .467 & 44.2 \\
\hline
\end{tabular}

of five trials for assessment of fish acceleration capacity with an SPC.

\section{Group Differences in Acceleration Performance} Measured with an SPC

Maximum acceleration for all groups of European sea bass was most frequently recorded in the first three intervals of the SPC of 2, 4, and $8 \mathrm{~cm}$, respectively (fig. 1). Although there is a certain degree of uncertainty regarding the start position with this method, it is quite plausible that the performances measured across these three intervals correspond to stage 2 of a fast start, the portion considered most reflective of escape success (Hale et al. 2002). Whether stage 3 (the first caudal fin undulation of continuous swimming) contributed to the acceleration measurements reported here is uncertain but unlikely given the relatively short distance and duration. Fish that were captured from the wild were significantly more likely to have their maximum accelerations recorded earlier in the SPC than cultured fish (not shown) and had significantly greater acceleration ability than all groups of cultured fish (fig. 4). Considering the minor differences in mass among these fish, these results are suggestive of wild fish being able to generate more force with their initial tail flips than conspecifics raised in culture, although differences in streamlining and speed of muscle activation could also be involved. Alternatively, behavioral differences between the groups may be responsible (see below).

Our results also show that the differences in maximal swimming speed between wild and cultured fish reported by Handelsman et al. (2010) could have arisen from significant differences in acceleration ability. Although the relationship does not appear strong (fig. 3), maximal sprint velocity in the 25$\mathrm{cm}$ intervals was a significant function of an individual's acceleration capacity $(P<0.001)$. Vanhooydonck et al. (2006) also found a significant relationship between acceleration and maximal sprint velocity in individual Anolis lizards. However, acceleration performance was not related to our measure of an animal's endurance capacity (critical swimming speed, $U_{\text {crit }}$; measured in cultured fish from the first release only; Claireaux et al. 2007). This is similar to the lack of relationship between $U_{\text {crit }}$ and maximal sprint swimming velocity in the SPC that we reported previously (Claireaux et al. 2007). Other studies using high-speed filming have also reported the relative independence of acceleration performance and endurance or aerobic performance in fish (e.g., Chappell and Odell 2004; Oufiero and Garland 2009).

\section{Repeatability and Variation}

The significant daily repeatability of acceleration performances (table 1; fig. 4) mirrored our earlier studies that established the daily repeatability of maximal sprint speed in the European sea bass but failed to show significant repeatability across 6 mo of residence in a simulated natural environment (Nelson and Claireaux 2005; Claireaux et al. 2007). Although fast-start and sprint swimming share many of the same component processes, they are morphologically and kinematically different. A fast start consists of an asymmetric movement of the fish body (Eaton et al. 2001), while a sprint involves a series (more than two) of rapid tail beats that are kinematically similar to those observed during sustained swimming (Domenici and Blake 1997). Our previous analyses of maximal sprint velocity (Nelson and Claireaux 2005; Claireaux et al. 2007) omitted any velocities recorded by the first four intervals of the SPC, so although those velocity values may have been manifestations of the accelerations reported here, they are completely independent measurements. Thus, the SPC is measuring two distinct modes of swimming: (1) a poorly defined beginning that involves rapid acceleration and most likely corresponds to stage 2 of a fast start and (2) sprint or burst and coast swimming that fish would typically employ in short pursuits, navigating strong currents, or avoiding trawls. Measured swimming velocities were actually higher in the SPC than in filmed fast-start determinations, as presumably positive accelerations accumulated while the fish tried to escape its pursuer (fig. 2). Although quite different in their temporal dimension, sprint and faststart responses have in common a strong behavioral component, which corresponds to information collection and processing and for which the main outcome is to devise an appropriate locomotor response (Domenici 2009; Marras et al. 2011). How individual fish appreciate a given environmental situation, and respond to it, has been shown to be highly variable (Domenici 2003; Jones and Godin 2010). This is demonstrated, for instance, by the reported interindividual variability of responsiveness, latency, reaction distance, and turning angle (Turesson et al. 2009; Marras et al. 2011). 
This variation in the behavioral component of the fast-start or sprinting responses may be responsible, at least in part, for the observed interindividual variability, as well as for the differences between fish from different origins. Behavioral studies have revealed that in a group of fish, individuals are readily identified as explorers or followers depending on their reaction when exposed to a novel environmental situation (Jones and Godin 2010). In our study, fish raised in culture were observed on occasion turning toward the source of stimulation, responding to the supposed predator as if they were looking for food. This was never observed in wild fish. It is quite likely that for wild fish, escaping a predator is more likely to influence survival than missing a meal. For farmed fish, on the other hand, the number one source of worry is likely to be competition with conspecifics for access to food and not the occurrence of predators. This is supported by the inability of the cultured fish to survive even $14 \mathrm{wk}$ in the mesocosms when exposed to avian predation (Handelsman et al. 2010). The extent to which these behavioral differences relate to mortality selection and underpin the phenotypic structure of experimental populations is un known, but it represents an interesting avenue for future research. The fact that the wild fish population was the only one to combine the highest acceleration and the highest maximal swimming speed in an SPC is certainly a good starting point.

The repeatability of locomotor performance over various timescales and environmental conditions has been documented in a variety of species, and evaluating repeatability has become standard practice in physiological ecology studies (e.g., van Berkum et al. 1989; Dohm 2002; Oufiero and Garland 2009; Marras et al. 2011). In fish, the short-term repeatability of sprint swimming performance is fairly well established (Nelson et al. 2002, 2008; Chappell and Odell 2004; Nelson and Claireaux 2005; Claireaux et al. 2007), but long-term repeatability of sprinting performance has been established only over relatively short periods in the laboratory for a few species (Reidy et al. 2000; Martínez et al. 2002; Nelson et al. 2008; Oufiero and Garland 2009). These laboratory studies have demonstrated stable sprint performance of Atlantic cod (Gadus morhua) over 1.5-3 mo, guppies (Poecilia reticulata) over 1-4 wk, and blacknose dace (Rhynichthys atratulus) over $7 \mathrm{~d}$ but not $10 \mathrm{wk}$. However, Nelson et al. (2008) present evidence that the lack of long-term repeatability of sprint performance in dace may be an artifact of laboratory residence because site-specific differences in sprint performance of wild-caught animals were stable over 1 yr (different individuals). Claireaux et al. (2007) report marginally stable sprint speed performances in sea bass that survived the 6-mo stay in mesocosms. Here we report that acceleration performance measured with an SPC was repeatable on a daily basis (table 1) but was not repeatable in animals that survived the 6-mo stay in the mesocosms. If one considers the great range of natural and imposed conditions experienced by these fish in the mesocosms (Claireaux et al. 2007), it is not surprising that we failed to detect repeatable acceleration performance. Fast-growing juveniles are subjected to a broad phenotypic reshaping that, coupled with rapidly changing dietary conditions (Claireaux et al. 2007), is liable to shuffle the between-individual hierarchy, similar to the lack of repeatable locomotor performance seen across metamorphosis in amphibians (Watkins 1997). Others have presented evidence suggesting that fish acceleration performance in the laboratory is repeatable on a short-term basis (e.g., Gibson and Johnston 1995; Fuiman and Cowan 2003); however, this area requires much more research on animals in the field or in mesocosm settings before extrapolations from laboratory results to the field can be given any credibility (Irschick et al. 2008). Daily repeatability of acceleration estimates in the same fish by highspeed cinematography and the SPC were virtually identical (table 1; fig. 2).

Effects of Size. There were no significant effects of size on acceleration performance within any of the four groups of sea bass. However, pooling of all the groups produced a data set with a larger size range that returned a significant inverse relation between body size and absolute acceleration performance, indicating that maximal acceleration scales as $M_{\mathrm{b}}^{-0.30}$ in juvenile sea bass. Because of the large interindividual variation in performance and the fact that this relationship predicts that a 200-g European sea bass would be unable to accelerate, we must consider this result provisional and valid only over the size range encompassed by our study. A meta-analysis by Vogel (2008) confirmed the general inverse scaling of acceleration in the aquatic medium, as did an earlier analysis by Domenici (2001). While our results with juvenile sea bass conform to this expectation, other studies have shown positive or no scaling of acceleration performance (e.g., Domenici and Blake 1997). We therefore predict that scaling of acceleration performance will be unique to each species and size class, ultimately depending on the changes affecting the morphology or the physiology of individuals as they grow.

Mesocosm Performance. The tidal earthen ponds used as mesocosms in this study provide a unique opportunity to illustrate the complexity of the processes involved in mortality selection and how they affect the trade-offs involved in environmental adaptation and the distribution of physiological traits within European sea bass populations. In the spring, trophic conditions are optimal and significant growth rates are generally observed. During that period, survival is essentially determined by tolerance to physicochemical stressors and disease. As summer progresses, however, food supply changes substantially as the abundance of the European sea bass's main prey (grass shrimp Palaemonetes varians, common prawn Palaemon serratus, and crangonid shrimp Philocheras triptinosus) declines. Under these conditions, a decrease in growth performance is generally observed, and the capacity to catch prey then becomes the main determinant of survivorship (G. Claireaux, personal communication). Both mesocosm releases reported here were during this mid- to late summer time period when prey capture ability should be critical.

Similar to sprint performance (Handelsman et al. 2010), there was no discernible advantage to being able to accelerate well while in the mesocosms under high-density, predator-free 
conditions. Intraspecific competition for limited forage, environmental changes, and disease are the presumed challenges faced by juvenile sea bass under these conditions, but neither survival nor growth could be predicted from an animal's acceleration ability measured before release to the mesocosms.

Allowing birds to prey on juvenile European sea bass in the simulated natural environments yielded some evidence that acceleration performance, measured in the laboratory, can contribute to ecological success. Wild fish were generally smaller than the cultured conspecifics that we compared them with (Handelsman et al. 2010), but even after correction for the aforementioned size effect (ANCOVA), wild fish could accelerate significantly faster (fig. 4). Thirty-five percent of these fish captured from the wild survived 14 wk of avian predation in the artificial estuaries, whereas no cultured fish did. There will be many factors besides sprint performance that differ between wild and cultured sea bass (Malavasi et al. 2004), and the wild fish that survived were not better accelerators than those that perished, although the sample sizes were small. So we can claim only very limited evidence from this study to support the idea that acceleration ability aids survival in this simulated field environment.

While the evidence that these differences in acceleration ability contributed to the differential survival of wild Dicentrarchus labrax after $14 \mathrm{wk}$ in the estuaries is not strong, that these significant differences exist after only 1-2 yr of differential ontogeny does not bode well for some potential applications for cultured fish. The numerous comparisons of swimming performance between wild and cultured fish tend to focus on longer-duration swimming tests (discussed in Handelsman et al. 2010) and tend to find that wild fish outperform cultured fish. Data on the differences between wild and cultured fish on the timescale of predator-prey interactions are much harder to find but generally favor wild fish performance. For example, Gibson and Johnston (1995) show a reduced maximal velocity achieved from an escape response of farmed juvenile turbot (Scophthalmus maximus) compared with wild juveniles but only if the wild fish were freshly captured, suggestive of a laboratoryinduced performance atrophy effect (Nelson et al. 2008). Walker et al. (2005) report diminished acceleration performance of domestic guppies (Poecilia reticulata) compared with wild conspecifics, which led to slightly greater vulnerability in staged predator encounters. In summary, while very few species have been examined, the results we report here as well as the literature suggest that the prognosis for cultured fish engaging in acceleration performances on par with their wild conspecifics is poor. While results from terrestrial studies suggest that in some natural systems, maximal performance levels are rarely used (Irschick 2003; but see Husak 2006), these results are not necessarily transferable to the denser, more viscous aquatic medium where locomotor performance is subject to different constraints and may be under different selection intensities.

Initial acceleration performance was not predictive of growth rates in the first release to the mesocosms (no predation), but when food resources in the estuaries were ample and fish densities were reduced as a result of mortalities in the previous intervals, a significant inverse relationship between growth rate in this last interval and acceleration performance measured after the fish were removed from the estuaries was observed (fig. 5). This apparent trade-off between growth and swimming performance was also observed for sprint performance in these fish and is discussed at length in Handelsman et al. (2010). An inverse relationship between growth and swimming performance has been reported by a number of other investigators (e.g., Billerbeck et al. 2001).

The final observation of note from the mesocosm experiments is that surviving cultured fish from the first release, despite being larger and undergoing a growth spurt (see above), generally (31/38) improved their acceleration performance after 6 mo in the estuaries. Because there was no significant effect of initial acceleration capacity on survival, this suggests that surviving the mesocosms entailed improvement of acceleration performance and not directional selection on existing performance. There are any number of speculative reasons for this result (better nutrition from natural foods, better water quality in the field, etc.), but we consider the most parsimonious explanation to be that animals that learned to accelerate better were able to better make the transition from food pellets to live prey and survived the mesocosms at the expense of those fish that did not.

\section{Acknowledgments}

We thank Jeff Klupt, Gaël Guillou, Michel Prineau, and Philippe Pineau for their valuable technical assistance. We also wish to thank Patrick Gentien for his help with various administrative aspects of this work. David McKenzie, Alex Shingles, Justin Meager, and Amber Marcum are also due thanks for assistance with fish collections. We also wish to acknowledge the financial support of Institut Français de Recherche pour l'Exploitation de la Mer (IFREMER), France (G.C.), the U.S. Department of State Fulbright Scholarship Board (J.A.N.), and Towson University and the U.S. National Science Foundation (DBI 9732442; J.A.N. and C.A.H.). Financial support by the European Union, Directorate Fisheries, through contract QLRS2002-00799, Project ETHOFISH, is acknowledged. This study was approved by Towson University Institutional Animal Care and Use Committee F9900RRR.08 and by Direction Générale de l'Alimentation, Sous Direction de la Santé et de la Protection Animales 006572.

\section{Literature Cited}

Adolph S.C. and T. Pickering. 2008. Estimating maximum performance: effects of intraindividual variation. J Exp Biol 211: 1336-1343.

Billerbeck J.M., T.E. Lankford, and D.O. Conover. 2001. Evolution of intrinsic growth and energy acquisition rates. I. Trade-offs with swimming performance in Menidia menidia. Evolution 55:1863-1872.

Blake R.W. and T.C. Law. 1996. Comparison of the fast-start 
performances of closely related, morphologically distinct threespine sticklebacks (Gasterosteus spp.). J Exp Biol 199: 2595-2604.

Brannon E.L., D.F. Amend, M.A. Cronin, J.E. Lannan, S. LaPatra, W.J. McNeil, R.E. Noble, et al. 2004. The controversy about salmon hatcheries. Fisheries 29:12-31.

Chappell M. and J. Odell. 2004. Predation intensity does not cause microevolutionary change in maximum speed or aerobic capacity in Trinidadian guppies (Poecilia reticulate $\mathrm{Pe}-$ ters). Physiol Biochem Zool 77:28-38.

Claireaux G., C. Handelsman, E. Standen, and J.A. Nelson. 2007. Thermal and temporal stability of swimming performance in the European sea bass. Physiol Biochem Zool 80: 186-196.

Dohm M.R. 2002. Repeatability estimates do not always set an upper limit to heritability. Funct Ecol 16:274-280.

Domenici P. 2001. The scaling of locomotor performance in predator-prey encounters: from fish to killer whales. Comp Biochem Physiol A 131:169-182.

- 2003. Habitat, body design and the swimming performance of fish. Pp. 137-160 in V.L. Bels, J.-P. Gasc, and A. Casinos, eds. Vertebrate biomechanics and evolution. BIOS Scientific, Oxford.

- 2009. Escape responses in fish: kinematics, performance and behavior. Pp. 123-170 in P. Domenici and B.G. Kapoor, eds. Fish locomotion: an eco-ethological perspective. Science, Enfield, NH.

Domenici P. and R.W. Blake. 1997. The kinematics and performance of fish fast-start swimming. J Exp Biol 200:11651178.

Eaton R.C., R.K.K. Lee, and M.B. Foreman. 2001. The Mauthner cell and other identified neurons of the brainstem escape network of fish. Prog Neurobiol 63:467-485.

Endler J.A. 1986. Natural selection in the wild. Princeton University Press, Princeton, NJ.

Falconer D.S. and T.F. McKay. 1996. Introduction to quantitative genetics. 4th ed. Longman, Harlow.

Fuiman L.A. and J.H. Cowan Jr. 2003. Behavior and recruitment success in fish larvae: repeatability and covariation of survival skills. Ecology 84:53-67.

Gibson S. and I.A. Johnston. 1995. Scaling relationships, individual variation and the influence of temperature on maximum swimming speed in early settled stages of the turbot Scophthalmus maximus. Mar Biol 121:401-408.

Hale M.E., J.H. Long, M.J. McHenry, and M.W. Westneat. 2002. Evolution of behavior and neural control of the fast-start escape response. Evolution 56:993-1007.

Handelsman C., G. Claireaux, and J.A. Nelson. 2010. Swimming ability and ecological performance of cultured and wild European sea bass (Dicentrarchus labrax) in coastal tidal ponds. Physiol Biochem Zool 83:435-445.

Husak J.F. 2006. Does survival depend on how fast you can run or how fast you do run? Funct Ecol 20:1080-1086.

Irschick D.J. 2003. Measuring performance in nature: implications for studies of fitness within populations. Integr Comp Biol 43:397-407.
Irschick D.J., J.J. Meyers, J.F. Husak, and J.F. Le Galliard. 2008. How does selection operate on whole-organism functional performance capacities? a review and synthesis. Evol Ecol Res 10:177-196.

Johnston I.A., J.L. Van Leeuwen, M.L.F. Davies, and T. Beddow. 1995. How fish power predation fast-starts. J Exp Biol 198: 1851-1861.

Jones K.A. and J.-G.J. Godin. 2010. Are fast explorers slow reactors? linking personality type and anti-predator behaviour. Proc R Soc B 277:625-632.

Kingsolver J.G., H.E. Hoekstra, J.M. Hoekstra, D. Berrigan, S.N. Vignieri, C.E. Hill, A. Hoang, P. Gibert, and P. Beerli. 2001 The strength of phenotypic selection in natural populations. Am Nat 157:245-261.

Lefrançois C. and P. Domenici. 2006. Locomotor kinematics and behavior in the escape response of European sea bass, Dicentrarchus labrax L., exposed to hypoxia. Mar Biol 149: 969-977.

Malavasi S., V. Georgalas, M. Lugli, P. Torricelli, and D. Mainardi. 2004. Differences in the pattern of antipredator behaviour between hatchery-reared and wild European sea bass juveniles. J Fish Biol 65A:143-155.

Marras S., G. Claireaux, D.J. McKenzie, and J.A. Nelson. 2010. Individual variation and repeatability in aerobic and anaerobic swimming performance of European sea bass, Dicentrarchus labrax. J Exp Biol 213:26-32.

Marras S., S.S. Killen, G. Claireaux, P. Domenici, and D.J. McKenzie. 2011. Behavioural and kinematic components of the fast-start escape response in fish: individual variation and temporal repeatability. J Exp Biol 214:3102-3110.

Martínez M., H. Guderley, J.A. Nelson, D. Webber, and J.-D. Dutil. 2002. Once a fast cod, always a fast cod: maintenance of performance hierarchies through changing food availability in cod (Gadus morhua). Physiol Biochem Zool 75:90100.

Nelson J.A. and G. Claireaux. 2005. Sprint swimming performance of juvenile European sea bass. Trans Am Fish Soc 134:1274-1284.

Nelson J.A., P.S. Gotwalt, S.P. Reidy, and D.M. Webber. 2002. Beyond $U_{\text {crit }}$ : matching swimming performance tests to the physiological ecology of the animal, including a new fish "drag strip." Comp Biochem Physiol A 133:289-302.

Nelson J.A., P.S. Gotwalt, C.A. Simonetti, and J.W. Snodgrass. 2008. Environmental correlates, plasticity and repeatability of differences in performance among blacknose dace (Rhinichthys atratulus) populations across a gradient of urbanization. Physiol Biochem Zool 81:25-42.

O'Steen S., A.J. Cullum, and A.F. Bennett. 2002. Rapid evolution of escape ability in Trinidadian guppies (Poecilia reticulata). Evolution 56:776-784.

O’Steen S., S.L. Eby, and J.A. Bunce. 2010. Dynamically honest displays: courtship locomotor performance indicates survival in guppies. Funct Ecol 24:1045-1053.

Oufiero C.E. and T. Garland Jr. 2009. Repeatability and correlation of swimming performances and size over varying 
time-scales in the guppy (Poecilia reticulata). Funct Ecol 23: 969-978.

Pickett G.D. and M.G. Pawson. 1994. Sea bass: biology, exploitation and conservation. Chapman \& Hall, London.

Reidy S.P., S.R. Kerr, and J.A. Nelson. 2000. Aerobic and anaerobic swimming performance of individual Atlantic cod. J Exp Biol 203:347-357.

Toledo-Guedes K., P. Sanchez-Jerez, J. Mora-Vidal, D. Girard, and A. Brito. 2011. Escaped introduced sea bass (Dicentrarchus labrax) infected by Sphaerospora testicularis (Myxozoa) reach maturity in coastal habitats off Canary Islands. Mar Ecol 2011:1-6.

Turesson H., A. Satta, and P. Domenici. 2009. Preparing for escape: anti-predator posture and fast-start performance in gobies. J Exp Biol 212:2925-2933.

Tytell E.D. and G.V. Lauder. 2008. Hydrodynamics of the escape response in bluegill sunfish, Lepomis macrochirus. J Exp Biol 211:3359-3369.

van Berkum F.H., R.B. Huey, J.S. Tsuji, and T. Garland Jr. 1989. Repeatability of individual differences in locomotor perfor- mance and body size during early ontogeny of the lizard Sceloporus occidentalis (Baird and Girard). Funct Ecol 3:97105.

Vanhooydonck B., A. Herrel, R. Van Damme, and D. Irschick. 2006. The quick and the fast: the evolution of acceleration capacity in Anolis lizards. Evolution 60:2137-2147.

Vogel S. 2008. Modes and scaling in aquatic locomotion. Integr Comp Biol 48:702-712.

Wakeling J.M. 2001. Biomechanics of fast-start swimming. Comp Biochem Physiol A 131:31-40.

Wakeling J.M. and I.A. Johnston. 1998. Muscle power output limits fast-start performance in fish. J Exp Biol 201:15051526.

Walker J.A., C.K. Ghalambor, O.L. Griset, D. McKenney, and D.N. Reznick. 2005. Do faster starts increase the probability of evading predators? Ecology 19:808-815.

Watkins T.B. 1997. The effect of metamorphosis on the repeatability of maximal locomotor performance in the Pacific tree frog Hyla regilla. J Exp Biol 200:2663-2668. 